\title{
Continuum description of finite-size particles advected by external flows: The effect of collisions
}

\author{
Cristóbal López ${ }^{1}$ and Andrea Puglisi ${ }^{2}$ \\ ${ }^{1}$ Instituto Mediterráneo de Estudios Avanzados (IMEDEA) (CSIC-UIB) and Departament de Fisica, Universidad de las Islas Baleares, \\ E-07071 Palma de Mallorca, Spain \\ ${ }^{2}$ INFM Center for Statistical Mechanics and Complexity and Dipartimento di Fisica, Università di Roma "La Sapienza," \\ Piazzale Aldo Moro 2, I-00185 Rome, Italy
}

(Received 4 July 2003; revised manuscript received 14 January 2004; published 28 April 2004)

\begin{abstract}
The equation of the density field of an assembly of macroscopic particles advected by a hydro-dynamic flow is derived from the microscopic description of the system. This equation allows one to recognize the role and the relative importance of the different microscopic processes implicit in the model: the driving of the external flow, the inertia of the particles, and the collisions among them. The validity of the density description is confirmed by comparisons of numerical studies of the continuum equation with direct simulation Monte Carlo simulations of hard disks advected by a chaotic flow. We show that the collisions have two competing roles: a dispersinglike effect and a clustering effect (even for elastic collisions). An unexpected feature is also observed in the system: the presence of collisions can reverse the effect of inertia, so that grains with lower inertia are more clusterized.
\end{abstract}

DOI: 10.1103/PhysRevE.69.046306

PACS number(s): 47.52. $+\mathrm{j}, 45.70 . \mathrm{Mg}$

\section{INTRODUCTION}

The transport of inertial particles in hydrodynamic flows has recently attracted the attention of many researchers [1]. The great number of applications of this topic, including, e.g., ecological problems (dynamics of plankton populations), geophysical processes (cloud formation, transport of pollutants in the atmosphere, or the oceans), or chemical engineering (chemical reactions in turbulent or chaotic flows), constitutes the main reason for this interest.

In most of these works, despite considering finite-size particles, the effect of the colliding processes among particles was completely disregarded. However, in some applications, as exemplified by rain initiation in clouds [2], the role of collisions seems to be crucial. Thus, in order to take them into account, in a recent paper [3] we have introduced a simple model of a granular material, $N$ particles subject to mutual collisions (elastic or inelastic), advected by a twodimensional chaotic flow (gravity is not considered). The model equivalently describes inertial particles colliding among them and immersed in a chaotic flow, with the density of the particles higher than that of the fluid. A novel result found in Ref. [3] is that collisions may strongly modify the scenario of the so-called preferential concentration [1] by which particles in turbulent flows, in the absence of collisions, tend to aggregate in specific spatial areas.

In this paper we derive from first principles the equation for the macroscopic density of grains (we indistinctly speak of grains or colliding particles) for the model of Ref. [3] (but with a flow not necessarily chaotic). Its properties are discussed and, in particular, we show how it can explain two different effects of collisions: dispersion and clustering. In addition, the macroscopic equation helps us to understand another surprising feature of the model, observed in the direct simulation Monte Carlo (DSMC) simulations, which we call reversed clustering: in the presence of collisions lower values of inertia can induce more aggregation of the particles.
The paper is organized as follows. In the following section we present our model and derive an explicit expression for the velocity of the grains. In Sec. III we obtain the evolution equation for the density field of particles. Next, in Sec. IV we present some of the most relevant features of this density equation; in particular, we discuss the relative importance of its different terms. In Sec. V we numerically check our theoretical results and, finally, in Sec. VI we summarize the paper.

\section{EQUATIONS OF MOTION AND STOCHASTIC TREATMENT OF COLLISIONS}

The equations of motion of $N$ granular particles driven by an external velocity field $\mathbf{u}(\mathbf{r}, t)$ are given by

$$
\begin{gathered}
\frac{d \mathbf{v}_{i}(t)}{d t}=-\frac{1}{\tau_{p}}\left[\mathbf{v}_{i}(t)-\mathbf{u}\left(\mathbf{r}_{i}(t), t\right)\right]+\gamma \sum_{j=1}^{N} \sum_{k}\left\{\left[\mathbf{v}_{i}(t)\right.\right. \\
\left.\left.-\mathbf{v}_{j}(t)\right] \cdot \hat{\mathbf{n}}_{i j}(t)\right\} \hat{\mathbf{n}}_{i j}(t) \delta\left(t-t_{i j}^{k}\right), \\
\frac{d \mathbf{r}_{i}(t)}{d t}=\mathbf{v}_{i}(t),
\end{gathered}
$$

where $i=1, \ldots, N, \mathbf{v}_{i}$ is the velocity of particle $i, \mathbf{r}_{i}$ its position, $\tau_{p}$ is the Stokes time, $\gamma=(1+r) / 2, r \in[0,1]$ is the restitution coefficient ( $r \leqslant 1$, with $r=1$ for elastic collisions), $\hat{\mathbf{n}}_{i j}(t)$ is the unitary vector joining the centers of particles $i$ and $j$ at time $t$ (in the following we will also use the notation $\hat{\mathbf{n}}\left(\mathbf{r}, \mathbf{r}^{\prime}\right)$ to denote a unitary vector joining vectors $\mathbf{r}$ and $\left.\mathbf{r}^{\prime}\right)$, $\delta$ is the Dirac delta, and with $t_{i j}^{k}$ we denote the times at which particles $i$ and $j$ make their $k$ th collision. The first term in Eq. (1) indicates the inertia of the particles and the second one shows how the velocity of a particle is modified because of the collisions with the rest. Without collisions Eqs. (1) and (2) are the equations of motion of $N$ spherical particles where the Bernoulli term, the added-mass term, the Basset- 
Boussinesq history integral term, and the Faxen corrections are neglected. Thus, the advection of the particles is modelized taking only the Stokes drag as the relevant term in the Maxey and Riley equations [4]. This is a consistent approximation when the particles of the granular system are much heavier than those of the fluid [5], which is the case that we are considering. It is also fundamental to mention that the relevant time scales of the problem were identified in Ref. [3] to be the typical time of the flow $T$, the Stokes time $\tau_{p}$, the mean collision time $\tau_{c}$, and, in the case of a chaotic flow, the time $\tau_{f}$, given by the inverse of the Lyapunov exponent of the flow.

In order to obtain a close expression for $\mathbf{v}_{i}(t)$ we first nondimensionalize time and velocities taking as unit scales the typical time $T$ and typical length $L$ of the flow, i.e., $t$ $\rightarrow t / T, u \rightarrow u T / L$, and $v \rightarrow v T / L$. Then $\mathbf{v}_{i}(t)$ will be obtained as a formal expansion in the nondimensional parameter $\tau_{p}$ $=\hat{\tau_{p}} / T$. From Eq. (1) one has straightforwardly

$$
\begin{aligned}
\mathbf{v}_{i}(t)= & \mathbf{u}\left(\mathbf{r}_{i}(t), t\right)-\tau_{p} d \mathbf{v}_{i} / d t,-\gamma \tau_{p} \sum_{i} \sum_{k}\left[\left(\mathbf{v}_{i}\right.\right. \\
& \left.\left.-\mathbf{v}_{j}\right) \cdot \hat{\mathbf{n}}_{i j}(t)\right] \hat{\mathbf{n}}_{i j}(t) \delta\left(t-t_{i j}^{k}\right),
\end{aligned}
$$

then this expression of $\mathbf{v}_{i}$ is itself substituted on the righthand side (rhs) of Eq. (3) and one obtains to order $\tau_{p}^{2}$,

$$
\begin{aligned}
\mathbf{v}_{i}(t)= & \mathbf{u}\left(\mathbf{r}_{i}(t), t\right)-\tau_{p} \frac{d \mathbf{u}}{d t}-\gamma \tau_{p} \sum_{i} \sum_{k}\left\{\left[\mathbf{u}\left(\mathbf{r}_{i}\right)\right.\right. \\
& \left.\left.-\mathbf{u}\left(\mathbf{r}_{j}\right)\right] \cdot \hat{\mathbf{n}}_{i j}(t)\right\} \hat{\mathbf{n}}_{i j}(t) \delta\left(t-t_{i j}^{k}\right)+\tau_{p}^{2} \hat{\boldsymbol{\eta}}_{i}(t)+\Theta\left(\tau_{p}^{3}\right) .
\end{aligned}
$$

If one writes down explicitly $\hat{\boldsymbol{\eta}}_{i}(t)$,

$$
\begin{aligned}
\hat{\boldsymbol{\eta}}_{i}(t)= & \frac{d^{2} \mathbf{u}\left(\mathbf{r}_{i}(t), t\right)}{d t^{2}}+\gamma \sum_{j} \sum_{k}\left(\frac{d}{d t}\left\{\left[\mathbf{u}\left(\mathbf{r}_{i}\right)-\mathbf{u}\left(\mathbf{r}_{j}\right)\right] \delta\left(t-t_{i j}^{k}\right)\right\}\right. \\
& \left.+\delta\left(t-t_{i j}^{k}\right) \frac{d}{d t}\left[\mathbf{u}\left(\mathbf{r}_{i}\right)-\mathbf{u}\left(\mathbf{r}_{j}\right)\right]\right)+\gamma^{2} \sum_{j} \sum_{k} \delta\left(t-t_{i j}^{k}\right) \\
& \times\left(\sum_{l} \sum_{m}\left(\mathbf{v}_{i}-\mathbf{v}_{l}\right) \delta\left(t-t_{i l}^{m}\right)-\sum_{n} \sum_{p}\left(\mathbf{v}_{j}-\mathbf{v}_{n}\right) \delta\left(t-t_{j n}^{p}\right)\right),
\end{aligned}
$$

one can see that it is a very complicated expression taking into account collisions among different particles. Neglecting the first term in Eq. (5) one can assume that it is some kind of effective stochastic term acting at the colliding times (this hypothesis will be checked in Sec. V), i.e., it denotes a random kick on a particle every time it collides:

$$
\hat{\boldsymbol{\eta}}_{i}(t)=\sum_{j=1}^{N} \sum_{k} \delta\left(t-t_{i j}^{k}\right) \zeta_{j}(t)
$$

where $\zeta_{j}(t)$ is a Gaussian white noise with zero mean and $\left\langle\zeta_{j}^{l}(t) \zeta_{k}^{m}\left(t^{\prime}\right)\right\rangle=2 D^{\prime \prime} \delta\left(t-t^{\prime}\right) \delta_{j k} \delta_{l m}$ (the superindex denotes vector component).

It is now easy to obtain that $\left\langle\hat{\boldsymbol{\eta}}_{i}(t)\right\rangle=0$, and the correlations

$$
\begin{aligned}
\left\langle\hat{\eta}_{i}^{k}(t) \hat{\eta}_{j}^{l}\left(t^{\prime}\right)\right\rangle & =2 D^{\prime \prime} \delta_{k l} \delta\left(t-t^{\prime}\right) \delta_{i j} \sum_{j} \sum_{m} \delta\left(t-t_{i j}^{m}\right) \\
& =2 D \delta_{i j} \delta_{k l} \delta\left(t-t^{\prime}\right) n\left(\mathbf{r}_{i}(t), t\right),
\end{aligned}
$$

where $n\left(\mathbf{r}_{i}(t), t\right)$ is such that $n(\mathbf{r}, t) d \mathbf{r} d t$ is the average number of collisions that a particle positioned in $\mathbf{r}$ undergoes at time $t . D^{\prime}$ and $D^{\prime \prime}$ are constants absorbed at the end in the definition of $D$, which is another constant of order $\sigma^{4} / T^{4}(\sigma$ is the diameter of the particles). This last statement comes from comparing the full expression at order $\tau_{p}^{2}$ in Eq. (5) with Eq. (7).

Finally, denoting $\hat{\boldsymbol{\eta}}_{i}(t)=\sqrt{\operatorname{Dn}\left(\mathbf{r}_{i}(t), t\right)} \boldsymbol{\eta}_{i}(t)$ we arrive to

$$
\begin{aligned}
\mathbf{v}_{i}(t)= & \mathbf{u}\left(\mathbf{r}_{i}(t), t\right)-\tau_{p} \frac{d \mathbf{u}}{d t}-\gamma \tau_{p} \sum_{i} \sum_{k}\left\{\left[\mathbf{u}\left(\mathbf{r}_{i}\right)\right.\right. \\
& \left.\left.-\mathbf{u}\left(\mathbf{r}_{j}\right)\right] \cdot \hat{\mathbf{n}}_{i j}(t)\right\} \hat{\mathbf{n}}_{i j}(t) \delta\left(t-t_{i j}^{k}\right)+\tau_{p}^{2} \sqrt{\operatorname{Dn}\left(\mathbf{r}_{i}(t), t\right)} \boldsymbol{\eta}_{i}(t) \\
& +\Theta\left(\tau_{p}^{3}\right),
\end{aligned}
$$

where $\boldsymbol{\eta}$ is a Gaussian white noise with zero mean and correlations $\left\langle\eta_{i}^{k}(t) \eta_{j}^{l}\left(t^{\prime}\right)\right\rangle=2 \delta_{k l} \delta_{i j} \delta\left(t-t^{\prime}\right)$.

At this point we make the hypothesis that Eq. (8) is valid for any $\tau_{p}$; in some sense, the higher-order terms $\Theta\left(\tau_{p}^{3}\right)$ just renormalize the diffusion coefficient. This is supported by the fact that for large $\tau_{p}$ the noise term in Eq. (8) dominates, which is consistent with the fact that in Eq. (1) for $\hat{\tau_{p}}$ large the dynamics of the particles is mainly driven by collisions. Thus, this hypothesis finally states that the net effect of the colliding processes is properly modelized by the noise term appearing in Eq. (8). This will also be checked in Sec. V.

\section{EVOLUTION EQUATION OF THE DENSITY FIELD}

Our aim is to obtain the evolution equation of the density field of particles. For this we closely follow [7,6] and define $\bar{\rho}(\mathbf{r}, t)=\sum_{i=1}^{N} \rho_{i}(\mathbf{r}, t) \equiv \Sigma_{i} \delta\left(\mathbf{r}_{i}(t)-\mathbf{r}\right)$. Using Ito calculus [7]

$$
\begin{aligned}
\frac{\partial \rho_{i}(\mathbf{r}, t)}{\partial t}= & -\boldsymbol{\nabla} \cdot\left[\rho_{i} \mathbf{u}(\mathbf{r}, t)\right]+\tau_{p} \boldsymbol{\nabla} \cdot\left(\rho_{i} \frac{d \mathbf{u}}{d t}\right) \\
& +\tau_{p}^{4} \frac{D}{2} \nabla^{2}\left[n(\mathbf{r}, t) \rho_{i}\right] \gamma \tau_{p} \boldsymbol{\nabla} \cdot\left[\rho_{i} \sum_{j=1}^{N} \sum_{k}\{[\mathbf{u}(\mathbf{r}, t)\right. \\
& \left.\left.\left.-\mathbf{u}\left(\mathbf{r}_{j}(t), t\right)\right] \cdot \hat{\mathbf{n}}\left(\mathbf{r}, \mathbf{r}_{j}\right)\right\} \hat{\mathbf{n}}\left(\mathbf{r}, \mathbf{r}_{j}\right) \delta\left(t-t_{i j}^{k}\right)\right] \\
& -\tau_{p}^{2} \sqrt{D} \boldsymbol{\nabla} \cdot\left[\rho_{i} \sqrt{n(\mathbf{r}, t)} \boldsymbol{\eta}_{i}(t)\right] .
\end{aligned}
$$

We assume now that the local free time between collisions, $\tau_{c}(\mathbf{r}, t)$, is everywhere smaller than any other characteristic time of the system, and that the mean free path $\lambda_{c}(\mathbf{r}, t)$ is also smaller than any other characteristic spatial scale. With these hypotheses we will proceed by integrating every term in Eq. (9) over small space-time cells $\Delta V \Delta t$ (with this integral normalized by this spatiotemporal volume) such that $\tau_{c}(\mathbf{r}, t)<\Delta t<\min \left\{\tau_{p}, 1, \tau_{f}\right\}$, and $\lambda_{c}^{2}(\mathbf{r}, t)<\Delta V<\lambda^{2}$, with $\lambda$ the minimum typical space scale of the system. Assuming that the fields $\bar{\rho}$ and $\mathbf{u}$ are constant over the above space-time 
cells, the integration over $\Delta V \Delta t$ (normalized with this volume) and the summation over index $i$ of the left-hand side (lhs) in Eq. (9), and of the first two terms on the rhs can be easily calculated.

More complicated are the other terms. Let us study them in detail beginning with the diffusion term [the third on the rhs of Eq. (9)]

$$
\begin{aligned}
\sum_{i} & \frac{1}{\Delta V \Delta t} \int_{t}^{t+\Delta t} d t^{\prime} \int_{\mathbf{r}}^{\mathbf{r}+\Delta V} d \mathbf{r}^{\prime} \frac{\tau_{p}^{4} D}{2} \nabla^{2}\left[\rho_{i}\left(\mathbf{r}^{\prime}, t^{\prime}\right) n\left(\mathbf{r}^{\prime}, t^{\prime}\right)\right] \\
& \approx \frac{\tau_{p}^{4} D}{2 \Delta V \Delta t} \sum_{i} \nabla^{2}\left[\rho_{i}(\mathbf{r}, t) \int d t^{\prime} d \mathbf{r}^{\prime} n\left(\mathbf{r}^{\prime}, t^{\prime}\right)\right] \\
& =\frac{\tau_{p}^{4} D}{2 \tau_{c}} \nabla^{2} \bar{\rho}(\mathbf{r}, t)^{2}
\end{aligned}
$$

The first equality arises from the assumption that $\rho_{i}$ is constant in $\Delta V \Delta t$, and in the last one we have used that $\int d t^{\prime} d \mathbf{r}^{\prime} n\left(\mathbf{r}^{\prime}, t^{\prime}\right) \approx \bar{\rho}(\mathbf{r}, t) \Delta V \Delta t / \tau_{c}$, which follows from the above-mentioned definition of the quantity $n(\mathbf{r}, t) d \mathbf{r} d t$. Note also that we have assumed that $\tau_{c}$ is constant but all our results can be extended to a $\tau_{c}$ dependent on the coordinates and time.

Next we proceed with the collision term, the fourth one on the rhs of Eq. (9). Using the notation $\mathbf{s}\left(\mathbf{r}^{\prime}, \mathbf{r}^{\prime \prime}, t^{\prime}\right)$ $\equiv\left(\left[\mathbf{u}\left(\mathbf{r}^{\prime}, t^{\prime}\right)-\mathbf{u}\left(\mathbf{r}^{\prime \prime}, t^{\prime}\right)\right] \cdot \hat{\mathbf{n}}\left(\mathbf{r}^{\prime}, \mathbf{r}^{\prime \prime}\right)\right) \hat{\mathbf{n}}\left(\mathbf{r}^{\prime}, \mathbf{r}^{\prime \prime}\right)$, and, again, that $\rho_{i}$ is approximately constant, the sum over $i$ and integration over $\Delta V \Delta t$ can be written as

$$
\begin{aligned}
&-\sum_{i} \frac{\gamma \tau_{p}}{\Delta V \Delta t} \int_{\Delta t} d t^{\prime} \int_{\Delta V} d \mathbf{r}^{\prime} \nabla_{\mathbf{r}^{\prime}} \\
& \cdot {\left[\rho_{i}\left(\mathbf{r}^{\prime}, t^{\prime}\right) \sum_{j, k} \mathbf{s}\left(\mathbf{r}^{\prime}, \mathbf{r}_{j}\left(t^{\prime}\right), t^{\prime}\right) \delta\left(t^{\prime}-t_{i j}^{k}\right)\right] } \\
& \approx-\sum_{i} \frac{\gamma \tau_{p}}{\Delta V \Delta t} \nabla_{\mathbf{r}} \cdot\left[\rho_{i}(\mathbf{r}, t) \int_{\Delta t} d t^{\prime}\right. \\
&\left.\quad \times \int_{\Delta V} d \mathbf{r}^{\prime} \sum_{j, k} \mathbf{s}\left(\mathbf{r}^{\prime}, \mathbf{r}_{j}\left(t^{\prime}\right), t^{\prime}\right) \delta\left(t^{\prime}-t_{i j}^{k}\right)\right] .
\end{aligned}
$$

Then, evaluating the time integral we obtain

$$
-\sum_{i} \frac{\gamma \tau_{p}}{\Delta V \Delta t} \boldsymbol{\nabla}_{\mathbf{r}} \cdot\left[\rho_{i}(\mathbf{r}, t) \int_{\Delta V} d \mathbf{r}^{\prime} \sum_{\langle j\rangle} \sum_{\langle k\rangle} \mathbf{s}\left(\mathbf{r}^{\prime}, \mathbf{r}_{j}\left(t_{i j}^{k}\right), t_{i j}^{k}\right)\right] .
$$

Here the $\Sigma_{\langle k\rangle}$ indicates a restriction in the sum to the colliding times $t_{i j}^{k}$ in the time interval $[t, t+\Delta t]$, and the notation $\Sigma_{\langle j\rangle}$ restricts the sum to the particles whose position is given by $\mathbf{r}_{j}\left(t_{i j}^{k}\right)=\mathbf{r}^{\prime}+\sigma \hat{\mathbf{n}}_{j}$, where, as already indicated, $\sigma$ is the diameter of the particles and $\hat{\mathbf{n}}_{j}$ is a unitary vector. This calculation deserves some clarifications: the restricted sum to the colliding times appears because of the $\delta$ function, and the sum restricted to the particles with the position as mentioned comes from the fact that, right at the collision event, two particles are separated by a distance $\sigma \hat{\mathbf{n}}$, where $\hat{\mathbf{n}}$ is an arbitrary unit vector.
Then we approximate $\quad \sum_{\langle k\rangle} \mathbf{s}\left(\mathbf{r}^{\prime}, \mathbf{r}_{j}\left(t_{i j}^{k}\right), t_{i j}^{k}\right)$ $\approx\left(\Delta t / \tau_{c}\right) \mathbf{s}\left(\mathbf{r}^{\prime}, \mathbf{r}_{j}(t), t\right)$ because $\Delta t / \tau_{c}$ is approximately the number of colliding events in the time interval $\Delta t$, and the sum over $\langle j\rangle$, with the help of the properties of the Dirac $\delta$ function, can be written as

$$
\begin{aligned}
\sum_{\langle j\rangle} \mathbf{s}\left(\mathbf{r}^{\prime}, \mathbf{r}_{j}(t), t\right)= & \sum_{j} \int_{|\overline{\mathbf{n}}|=1} d \overline{\mathbf{n}} \mathbf{s}\left(\mathbf{r}^{\prime}, \mathbf{r}^{\prime}+\sigma \overline{\mathbf{n}}, t\right) \delta\left(\mathbf{r}_{j}(t)-\left(\mathbf{r}^{\prime}\right.\right. \\
& +\sigma \overline{\mathbf{n}})) \sigma,
\end{aligned}
$$

so that now we do not have to manage with a restricted sum. Moreover, the sum of the $\delta$ s is the definition of $\bar{\rho}$, and we have that Eq. (13) is just

$$
\begin{aligned}
& \sigma \int_{|\overline{\mathbf{n}}|=1} d \overline{\mathbf{n}} \mathbf{s}\left(\mathbf{r}^{\prime}, \mathbf{r}^{\prime}+\sigma \overline{\mathbf{n}}, t\right) \bar{\rho}\left(\mathbf{r}^{\prime}+\sigma \overline{\mathbf{n}}\right) \\
& \quad=\sigma^{2} \int_{|\bar{n}|=1} d \overline{\mathbf{n}}\left\{\left[\overline{\mathbf{n}} \cdot \nabla_{\mathbf{r}^{\prime}} \mathbf{u}\left(\mathbf{r}^{\prime}, t\right)\right] \cdot \overline{\mathbf{n}}\right\} \overline{\mathbf{n}} \bar{\rho}\left(\mathbf{r}^{\prime}, t\right)+\Theta\left(\sigma^{3}\right) ;
\end{aligned}
$$

the equality comes from considering a Taylor expansion in powers of $\sigma$. Therefore, the collision term takes the form (assuming again constancy of the density and velocity fields in $\Delta V$ )

$$
-\frac{\gamma \sigma^{2} \tau_{p}}{\tau_{c}} \nabla_{\mathbf{r}} \cdot\left[\bar{\rho}(\mathbf{r}, t)^{2} \int_{|\overline{\mathbf{n}}|=1} d \overline{\mathbf{n}}\left[\left(\overline{\mathbf{n}} \cdot \nabla_{\mathbf{r}} \mathbf{u}(\mathbf{r}, t) \cdot \overline{\mathbf{n}}\right] \overline{\mathbf{n}}\right] .\right.
$$

Finally, taking the average over the noise as in Ref. [6], the last term in Eq. (9) disappears, and noting $\rho(\mathbf{r}, t)$ $\equiv\langle\bar{\rho}(\mathbf{r}, t)\rangle$ we obtain the evolution equation for this density field [8]

$$
\begin{aligned}
\frac{\partial \rho(\mathbf{r}, t)}{\partial t}= & -\boldsymbol{\nabla} \cdot[\rho \mathbf{u}(\mathbf{r}, t)]+\tau_{p} \boldsymbol{\nabla} \cdot\left(\rho \frac{d \mathbf{u}}{d t}\right)+\frac{\tau_{p}^{4} D}{2 \tau_{c}} \nabla^{2}\left(\rho^{2}\right) \\
& -\frac{\gamma \sigma^{2} \tau_{p}}{\tau_{c}} \nabla_{\mathbf{r}} \cdot\left[\bar{\rho}^{2} \int_{|\overline{\mathbf{n}}|=1} d \overline{\mathbf{n}}\left\{\left[\overline{\mathbf{n}} \cdot \nabla_{\mathbf{r}} \mathbf{u}(\mathbf{r}, t)\right] \cdot \overline{\mathbf{n}}\right\} \overline{\mathbf{n}}\right] .
\end{aligned}
$$

Here we have used the approximation $\left\langle\bar{\rho}^{2}\right\rangle \approx\langle\bar{\rho}\rangle^{2}$, since we expect that the densities in a point of space and time are likely to be uncorrelated for different realizations of the noise.

It is very important to clarify the meaning of the noise average performed to obtain the $\rho$ field. This has been done following the arguments of Ref. [6]. In our case, taken the average over the noise essentially means smoothing out the higher-order corrections to the velocity of the particles coming from complicated collision processes that take place in the time interval $\Delta t$. 


\section{RELATIVE IMPORTANCE OF THE TERMS IN THE DENSITY EQUATION, AND REVERSED CLUSTERING}

Some relevant features of Eq. (16) come immediately to light. First, the inelastic character (the value of $\gamma$ ) of the collisions is almost irrelevant at this level of description. The presence of the external driving flow turns negligible the difference between elastic and inelastic colliding processes. Also, the collision-induced diffusivity is, as expected, dependent on the density of particles. This is clearly recognized by rewriting it as $\left(\tau_{p}^{4} D / 2 \tau_{c}\right) \nabla^{2}\left(\rho^{2}\right)=\left(\tau_{p}^{4} D / \tau_{c}\right) \nabla(\rho \nabla \rho)$ $\equiv \boldsymbol{\nabla}(\hat{D}(\rho) \nabla \rho)$, where we have defined $\hat{D}(\rho)=\left(\tau_{p}^{4} D / 2 \tau_{c}\right) \rho$.

Most of the results of the discrete model (1) and (2) that were presented in Ref. [3] can be understood in the framework of Eq. (16). Let us put a label on each term on the rhs of Eq. (16): the first is the pure advective term, which we label with the letter $a$, the second is the inertial term $b$ (of order $\tau_{p}$ ), which is responsible for preferential concentration in the absence of collisions [1], the third is a collisioninduced diffusive term $c$ of order $\tau_{p}^{4} D / \tau_{c}$, and the last is a collision-induced term $d$ of order $\tau_{p} \sigma^{2} / \tau_{c}$, which could also induce clustering.

In the absence of collisions $\left(\tau_{c} \rightarrow \infty\right)$ the last two terms vanish and there is only competition between the advection $a$ and the clusterizing inertial term $b$, e.g., when $\tau_{p}>\sim 1$ it appears that inertia dominates and the system is strongly clusterized. On the other side, in the limit $\tau_{p} \rightarrow 0$ ( $b$ vanishes), it is the $d$ term that can induce clustering (when $\tau_{c}$ $\ll \tau_{p}$ ); however the term $c$ may eventually become stronger than $d$ giving rise to a homogeneous distribution of particles. The unexpected feature emerging from this analysis is that the diffusive homogenizing term $c$ dominates at high values of $\tau_{p}$, thus reversing the effect of inertia that, in collisionless systems, enhances concentration. In the presence of collisions, therefore, we have a reversed clustering phenomenon: for small values of $\tau_{p}$, i.e., inertia, the density is more clusterized than at larger values.

\section{NUMERICAL SIMULATIONS}

We have checked numerically our system in two spatial dimensions by means of DSMC simulations of the hard disk model (similar to those performed in Ref. [3]) and by a numerical study (a Lax integration scheme) of Eq. (16). The DSMC is a well established algorithm [9] that allows one to simulate gaseous systems in a rapid and efficient way, with the assumption of negligible correlations at short range. We have used a variant of DSMC that takes into account the Enskog factor due to high density corrections, in order to obtain more accurate simulations of the clusterized situations. In Appendix we give details about this simulation scheme. First of all, we have verified the stochastic approximation done in Eq. (8), using a simple velocity field given by $u_{x}\left(\mathbf{r}_{i}(t), t\right)=U \cos \left[2 \pi y_{i}(t) / L\right], u_{y}=0$, with $\mathbf{r}_{i}(t)=\left[x_{i}(t), y_{i}(t)\right]$. We calculate the quantity given by the lhs of Eq. (8) minus the first three terms on the rhs, cumulated for a little time period $\overline{\Delta t}$ (greater than the simulation time step, but shorter than the mean free time) and divided by $\tau_{p}^{2} \sqrt{n}$. We have per-

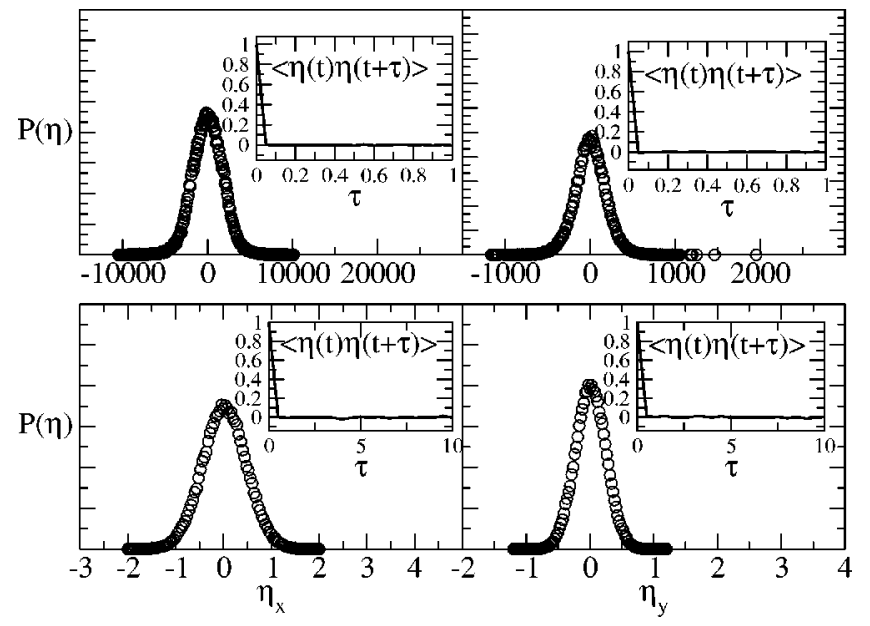

FIG. 1. Distributions of the residual term (left, $x$ component; right, $y$ component) of Eq. (8) which is assumed to be a Gaussian white noise. The form of the distribution and the self-correlation (displayed in the insets) confirm this assumption. The flow used in this simulation is a sinusoidal shear, i.e., $u_{x}\left(\mathbf{r}_{i}(t), t\right)$ $=U \cos \left[2 \pi y_{i}(t) / L\right], u_{y}=0$, where $\mathbf{r}_{i}(t)=\left[x_{i}(t), y_{i}(t)\right]$. For the upper plot (large $\tau_{p}$ ) the time step used in the DSMC is $\Delta t=0.0001$, size of the DSMC cells is $0.6 \times 0.6$, mean free time is 0.01 , mean free path 5, length of the time period used to calculate $\eta$ is $\overline{\Delta t}=0.005$, $\hat{\tau_{p}}=1, \quad r=1, N=L^{2}=1000, \sigma=0.3$, and $U=10000$ so that $\tau_{p}$ $=U \hat{\tau_{p}} / L \sim 300$. For the bottom plot (small $\left.\tau_{p}\right) \Delta t=0.01$, DSMC cells are $2 \times 2$, mean free time is 0.6 , mean free path $18, \overline{\Delta t}=0.5$, $\hat{\tau_{p}}=1, r=1, N=L^{2}=10000, \sigma=0.6$, and $U=50$ so that $\tau_{p}=0.5$.

formed this for both cases: large and small $\tau_{p}$, in order to check also the discussion below Eq. (8). One can appreciate in Fig. 1 that in the two cases this quantity is a Gaussian white noise.

Typical patterns for the distribution of particles obtained with DSMC at large time can be seen in Fig. 2. The flow we have used here is the cellular flow derived from the streamfunction [10] $\quad \psi(x, y, t)=U \sin (2 \pi / L[x$ $\left.\left.+B_{0} \cos (\omega t)\right]\right) \sin (2 \pi / L y)$, with $B_{0}$ and $\omega$ the amplitude and frequency of the temporal perturbation, respectively. In this figure we just show a small part, around $2 \%$ of the entire system, in order to better appreciate the clustering areas. For the sake of clarity we also show, in red lines, the streamlines of the flow for $B_{0}=0$. We have chosen two opposite situations. In Figs. 2(a) and 2(b) we have studied a strong inertia case $\left(\tau_{p}>1\right)$ with the panel (b) corresponding to the same case as (a) but without collisions. Note that in the absence of collisions $b>a$ and preferential concentration can be recognized [panel (b)], while with (elastic) collisions we have that $c \gg d \gg b \gg a$ so that diffusion dominates and the distribution of particles in (a) is homogeneous. Thus, in this case we observe the dispersing effect of the collisions. In Figs. 2(c) and 2(d), instead, we have considered a weak inertia case, $\tau_{p}<1$, where Fig. 2(d) is the case with no collisions. Now, without collisions we have that $a>b$ and the flow homogenizes the distribution of grains [see Fig. 2(d)]. With collisions, instead, we have $d>a>b>c$ and collision induced clustering is observed [Fig. 2(c)]. Therefore, this case shows the clustering effect of the collisions. 

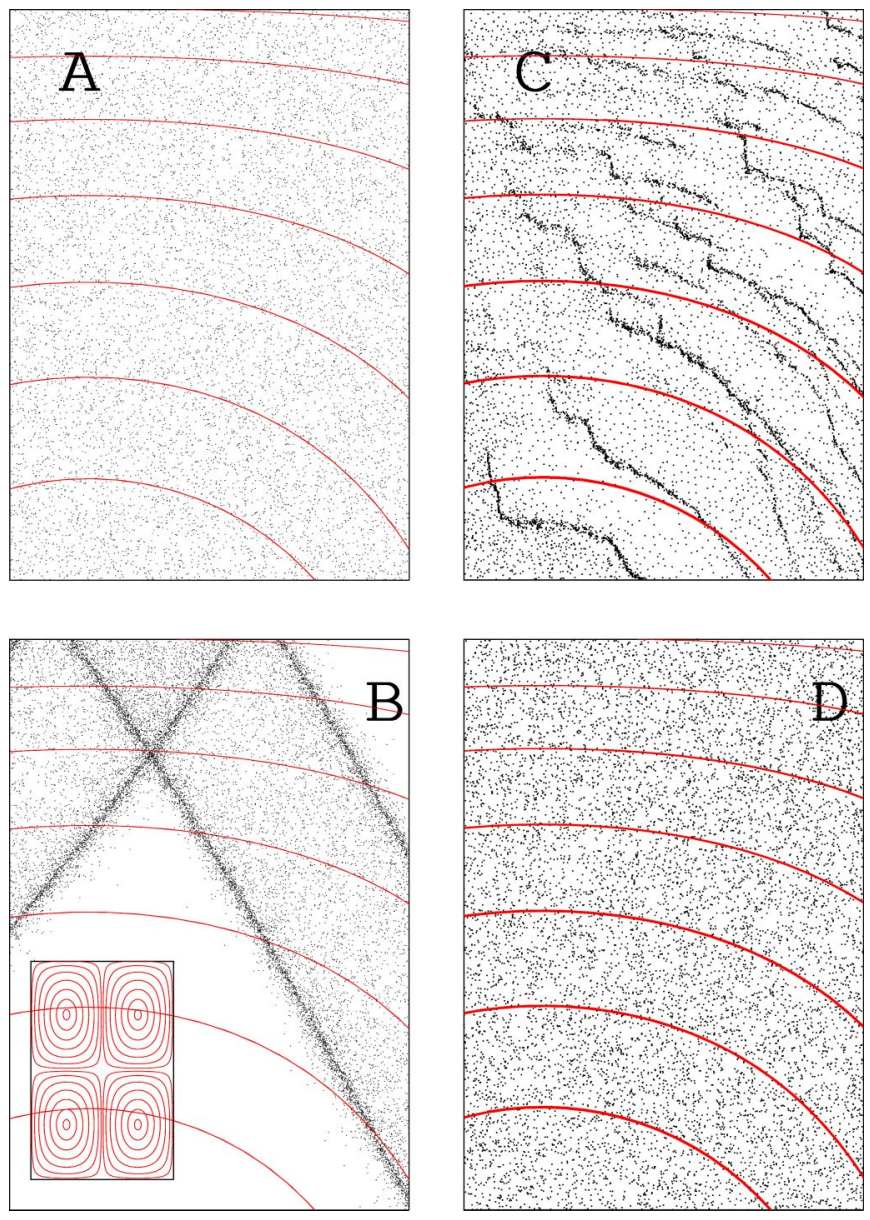

FIG. 2. (Color online). Instantaneous density patterns, obtained with DSMC, for the $2 d$ cellular flow, $N=500000$ and $L=\sqrt{N}$. Panels (b) and (d) are without collisions. Frames (a) and (b): $U=30, B_{0}$ $=1 \omega=1\left(\tau_{f} \sim 0.4\right), \hat{\tau_{p}}=10, \sigma=0.3, r=1, \tau_{c} \sim 0.02$ [only for (a)]. Panels (c) and (d): $U=110, B_{0}=1, \omega=10\left(\tau_{f} \sim 0.3\right), \tau_{p}=0.003, \sigma=0.5$, $r=1, \tau_{c} \sim 0.0002$ [for (c)]. come from DSMC. Note that a small part $(\sim 2 \%)$ of the whole system is potrayed. Solid (red) lines represent streamlines of the flux with $B_{0}=0$. The inset in (b) shows these for the entire spatial domain.

The numerical comparison of the DSMC with the continuum equation is shown in Fig. 3. Here we measure the $P_{M}(n)$ function, which gives the histogram (normalized to unity) of the number of boxes containing $n$ particles after dividing the system in $M$ boxes. Note that as the clustering is stronger the deviations of $P_{M}(n)$ from a Poissonian (homogeneous distribution) are more evident. On the right we plot the function for the DSMC patterns of Figs. 2(a) and 2(b) (upper right panel) and Figs. 2(c) and 2(d) (lower right). On the left, we show $P_{M}(n)$ for the distribution calculated with the continuum equation and the same value of the parameters. Note that now a homogeneous distribution of particles corresponds to a constant density and that is the reason why in this case $P_{M}(n)$ resembles a Dirac $\delta$ distribution centered around this constant value.

The results shown in Figs. 2 and 3 clearly indicate the relevant role of the collisions for inertial particles immersed in a flow, verifying as well the important and unexpected

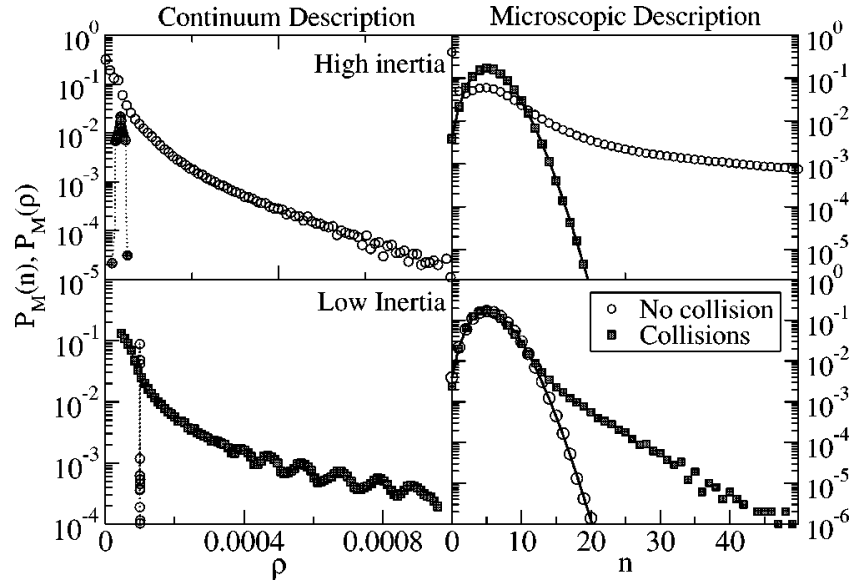

FIG. 3. $P_{M}(n)$ function for the distribution of particles obtained from DSMC (right panels) and for the density of particles from the continuum equation (16) (left) for the same parameter values. Upper right are the cases in Figs. 2(a) and 2(b), that is, with high inertia, and the lower right corresponds to the Figs. 2(c) and 2(d) panels (low inertia).

phenomenon of reversed clustering that appears on the basis of the inspection of terms $c$ and $d$ of Eq. (16). Briefly, in the absence of collisions particles with inertia tend to aggregate, and the largest is the value of $\tau_{p}$ the more compact is the aggregation of particles [1]. This can be seen comparing Figs. 2(b) and 2(d). However, when collisions are taken into account this last statement can be wrong, and just the contrary occurs: inertia is decreasing but aggregation increases [again this is what happens if we compare Figs. 2(a) and 2(c)]. Note that in these last two cases we have that $\tau_{c}<\tau_{p}$ so that collisions dominate the dynamics. Equation (16) perfectly reflects this situation: the main difference between the two cases is originated by the value of $\tau_{p}$ that influences the relative importance of terms $c$ and $d$, as well as the relative importance between $a$ and $b$ in the absence of collisions.

It is important to note that this effect may also appear in segregation processes, i.e., in systems with particles of different sizes or densities. Obviously, these particles have different values of $\tau_{p}$ and the same scenario, just commented, emerges. Finally we put in evidence that the inelasticity of collisions (typical of granular materials) here just plays the role of slightly enhance spatial correlations, i.e., clustering. This is not taken into account by Eq. (16) but can be observed in the DSMC simulations.

\section{SUMMARY}

The evolution of the density field of a large number of colliding finite-size particles driven by an external flow has been derived. It is important to note that no premise has been performed on the flow so our results are equally valid for laminar, chaotic, or turbulent flows. However, in the numerics we have used a chaotic flow just for its interest and simplicity, and also to compare with the results of our previous work [3]. In this equation one can recognize two, in principle, competing roles of the collisions: clustering and dispersion. The relative strengths of the important time scales 
involved in the system will finally determine if the particles tend to cluster or, on the contrary, are uniformly distributed in the space. Collisions may even invert the typical scenario found for inertial particles, showing the reversed clustering phenomenon. We have also presented a numerical study of the continuum equation and compared with DSMC simulations of the system of discrete particles. The good agreement gives support to our theory.

\section{ACKNOWLEDGMENTS}

We acknowledge fruitful discussions with Umberto Marini Bettolo Marconi. C.L. acknowledges support from MCyT of Spain under Project No. REN2001-0802-C02-01/ MAR (IMAGEN).

\section{APPENDIX: THE DSMC}

Direct simulation Monte Carlo, also known as Bird Method [9], is a simulation scheme used to solve Boltzmann equations. With some conditions and in well defined limits its results are proved to converge to the solution of the Boltzmann equation for a gas of hard spheres [11]. The algorithm consists of two main steps: (a) the free flow and (b) the collisions. At every time step of length $\Delta t$ (lesser than any characteristic physical time, e.g., the collision time) all the particles are moved freely, i.e., disregarding possible collisions and then the collision procedure is applied: the system is divided into cells of linear size lesser than any characteristic physical length (e.g., the mean free path) and particles in the same cell may collide randomly. For each cell $c$ a number $m_{c}$ of couples of particles is chosen with probability proportional to their scattering section, i.e., in this case proportional to their relative velocities. The number $m_{c}$ is calculated as $m_{c}=\omega_{c} \Delta t$, where $\omega_{c}$ is the average collision frequency estimated in the cell $c$, by assuming a Gaussian distribution of velocities with variance given by the actual variance of velocities of the particles inside the cell. As is known, the Boltzmann equation is a correct description of a gas of hard particles only in the dilute limit $N \rightarrow \infty, \sigma \rightarrow 0$ with finite $N \sigma^{d-1}$ ( $d$ the space dimension). When the gas cannot be considered dilute, correlations arise in the form of an enhancement of the collision frequency and in complicated excluded volume effects. It is accepted that at not too high packing fraction, the so-called Enskog correction to the Boltzmann equation gives a sufficient description of these effects. In the Boltzmann equation this correction appears as a simple multiplicative term in front of the collision integral, which is equivalent to an increase of the collision frequency. The Enskog correction is usually taken to be the static correlation function $g(r)$ evaluated at contact, i.e., $r=\sigma$, for which approximated forms [dependent upon the local volume fraction $\phi=N_{c} \pi(\sigma / 2)^{2} / V_{c}$, with $V_{c}$ the area of a cell and $N_{c}$ the number of particles in the cell] are available. We (in $d=2$ ) have used the following form [12]:

$$
g(\sigma)=\frac{1-7 \phi / 16}{(1-\phi)^{2}} .
$$

[1] T. Elperin, N. Kleeorin, and I. Rogachevskii, Phys. Rev. Lett. 77, 5373 (1996); E. Balkovsky, G. Falkovich, and A. Fouxon, ibid. 86, 2790 (2001); T. Nishikawa, Z. Toroczkai, C. Grebogi, and T. Tél, Phys. Rev. E 65, 026216 (2001); C. López, ibid. 66, 027202 (2002); I. J. Benczik, Z. Toroczkai, and T. Tél, ibid. 67, 036303 (2003).

[2] G. Falkovich, A. Fouxon, and M. G. Stepanov, Nature (London) 419, 151 (2002).

[3] C. López and A. Puglisi, Phys. Rev. E 67, 041302 (2003).

[4] M. R. Maxey and J. J. Riley, Phys. Fluids 26, 883 (1983).

[5] M. R. Maxey, J. Fluid Mech. 174, 441 (1987).

[6] U. M.B. Marconi and P. Tarazona, J. Chem. Phys. 110, 8032
(1999).

[7] D. S. Dean, J. Phys. A 29, L613 (1996).

[8] In two spatial dimensions the integral in the collision term can be performed and one obtains $-\left(\gamma \sigma^{2} \tau_{p} \pi / 4 \tau_{c}\right)\left(\partial_{x}\left[\rho^{2}\left(\partial_{x} u_{y}\right.\right.\right.$ $\left.\left.+\partial_{y} u_{x}\right)+\partial_{y}\left[\rho^{2}\left(\partial_{x} u_{x}-\partial_{y} u_{y}\right)\right]\right)$.

[9] G. A. Bird, Molecular Gas Dynamics (Clarendon Press, Oxford, 1976).

[10] T. H. Solomon and J. P. Gollub, Phys. Rev. A 38, 6280 (1988).

[11] C. Cercignani, R. Illner, and P. Pulvirenti, The Mathematical Theory of Dilute Gases (Springer-Verlag, New York, 1994).

[12] W. F. Carnahan and K. E. Starling, J. Chem. Phys. 51, 635 (1969). 